\title{
Minimal Representation of Convex Polyhedral Sets ${ }^{1}$
}

\author{
J. TELGEN ${ }^{2}$ \\ Communicated by O. L. Mangasarian
}

\begin{abstract}
A system of linear inequality and equality constraints determines a convex polyhedral set of feasible solutions $S$. We consider the relation of all individual constraints to $S$, paying special attention to redundancy and implicit equalities. The main theorem derived here states that the total number of constraints together determining $S$ is minimal if and only if the system contains no redundant constraints and/or implicit equalities. It is shown that the existing theory on the representation of convex polyhedral sets is a special case of the theory developed here.
\end{abstract}

Key Words. Minimal representation, systems of linear constraints, linear inequalities, linear equalities.

\section{Introduction}

Systems of linear equality constraints have been studied extensively in the past, but systems of linear inequality constraints elicited virtually no interest until the advent of game theory in 1944 and linear programming in 1947. However, after the formulation of many practical problems as linear programming problems and the development of the simplex method by Dantzig (Ref. 2), a widespread interest in systems of linear constraints arose.

Only recently, the emphasis in these studies has shifted from the system as a whole to the individual constraints. Until the early sixties, systems of linear equalities and inequalities were studied from a system point of view,

\footnotetext{
${ }^{1}$ The author is indebted to Dr. A. C. F. Vorst (Erasmus University, Rotterdam, Holland) for stimulating discussions and comments, which led to considerable improvements in many proofs. Most of the material in this paper originally appeared in the author's dissertation (Ref. 1). The present form was prepared with partial support from a NATO Science Fellowship for the Netherlands Organization for the Advancement of Pure Research (ZWO) and a CORE Research Fellowship.
} 
in the sense that the system was more important than the individual constraints. A number of interesting results were derived for the solvability and the geometric properties of a system of linear constraints without considering the constraints individually (Refs. 3-6).

From the early sixties on, a number of papers were published treating the subject from a constraint point of view, in the sense that more attention is paid to the individual constraints within the system. As a consequence, for example, redundancy, which is a phenomenon typically related to individual constraints within a system, was taken into consideration. As a witness, the first paper entirely devoted to redundancy was written in 1962 by Boot (Ref. 7). Other papers examplifying this approach are Refs. 8-10.

Here, we combine both approaches to derive a minimality property for general systems of linear constraints. We consider a system of both equality and inequality constraints in the form

$$
A x=a, \quad B x \leqslant b,
$$

with

$$
A \in R^{m_{a} \times n}, \quad a \in R^{m_{a}}, \quad B \in R^{m_{b} \times n}, \quad b \in R^{m_{b}}, \quad x \in R^{n} .
$$

The rows of $A$ and $B$ are denoted by $A_{i}$ and $B_{i}$, respectively. The feasible region corresponding to the system (1) is denoted as

$$
S=\left\{x \in R^{n} \mid A x=a, B x \leqslant b\right\} .
$$

Throughout this paper, it is assumed that there exists a feasible solution for (1), i.e.,

$$
S \neq \varnothing .
$$

Redundant constraints can be omitted from the system (1) without changing $S$. In Section 2 , we consider both redundant inequality constraints and redundant equality constraints in some detail.

Implicit equalities are inequality constraints that are satisfied as an equality in all solutions $x \in S$. Therefore, they may be replaced by (explicit) equality constraints without changing $S$. We elaborate on the topic of implicit equalities in Section 3 and develop some interesting relations between redundant constraints and implicit equalities.

In Section 4 , we introduce the concept of a minimal representation of a convex polyhedral set. Then, we proceed to prove the main theorem, which asserts that a system of linear constraints is a minimal representation if and only if the system contains no redundant constraints and no implicit equalities.

We conclude with a section on existing theory, in which it is shown that our result is a generalization of the theory as given by Shefi, Luenberger, and Eckhardt (Refs. 11-13). 


\section{Redundancy}

Redundant constraints in the system (1) do not play a role in determining the feasible region $S$ : they can be omitted without changing $S$. To define redundant constraints more formally, we denote, for fixed $k$ :

$$
\begin{gathered}
S^{k}=\left\{x \in R^{n} \mid A_{i} x=a_{i}, \forall i \neq k, B x \leqslant b,\right\}, \\
S_{k}=\left\{x \in R^{n} \mid A x=a, B_{i} x \leqslant b_{i}, \forall i \neq k\right\} .
\end{gathered}
$$

Definition 2.1. The constraint $A_{k} x=a_{k}, 1 \leqslant k \leqslant m_{a}$, is a redundant equality constraint in the system (1) if and only if

$$
S^{k}=S \text {. }
$$

Definition 2.2. The constraint $B_{k} x \leqslant b_{k}, 1 \leqslant k \leqslant m_{b}$, is a redundant inequality constraint in the system (1) if and only if

$$
S_{k}=S \text {. }
$$

In these definitions, it should be noted that there is a difference between identifying a constraint as being redundant and removing that constraint. Since the concept of redundancy is defined relative to the system (1), removing one redundant constraint changes the system and may cause other (originally) redundant constraints to become nonredundant.

Although the definition of redundant constraints is not generally agreed upon in the literature, the topic has been studied quite extensively in the last two decades. Papers mainly considering the theory are Refs. 8 and 11-14; methods to identify redundant constraints are presented in Refs. $1,7,9$ and 15-21. A good deal of this work is motivated by the possible use of these techniques in mathematical programming algorithms and the expected gains, both in terms of computations and in terms of knowledge, in some applications (Refs. 15 and 21-24). A survey of these developments, together with extensive computational experience, is given in Ref. 25.

The redundancy of an equality constraint is related to the rank of the matrix $A$, denoted by $r(A)$, by the following well-known theorem.

Theorem 2.1. The constraint $A_{k} x=a_{k}$ is redundant in the system (1) if $r(A)$ does not change by removing $A_{k}$ from $A$.

As a direct consequence, we have the following corollary.

Corollary 2.1. The system (1) contains at least one redundant equality constraint if

$$
r(A)<m_{a} \text {. }
$$


The converse of the corollary is obviously not true, since any redundant equality may be replaced by two inequalities such as to make $r(a)=m_{a}$. Only under some additional assumptions can it be proved that the system (1) contains redundant equality constraints if and only if $r(A)<m_{a}$ (Theorem 3.3). Furthermore, note that it is not necessarily true that every equality constraint is redundant if $r(A)<m_{a}$.

Next, we define

$$
u_{k}(x)=b_{k}-B_{k} x
$$

Then, it follows from Definition 2.3 that

$$
B_{k} x \leqslant b_{k}, \quad 1 \leqslant k \leqslant m_{b},
$$

is a redundant inequality if and only if

$$
\hat{u}_{k}=\min \left\{u_{k}(x) \mid x \in S_{k}\right\} \geqslant 0 .
$$

To see this, note that

$$
S_{k}=S
$$

if and only if

$$
B_{k} x \leqslant b_{k}, \quad \forall x \in S_{k},
$$

which is equivalent to (2).

If $\hat{u}_{k}=0$, then the inequality is termed weakly redundant; if $\hat{u}_{k}>0$, it is termed strongly redundant. Unless explicitly mentioned otherwise, the term "redundant" will be used to indicate constraints that are either strongly or weakly redundant. A redundant inequality constraint is related to the feasibility of a system of linear constraints by the turnover lemma.

Lemma 2.1. (Turnover Lemma, Ref. 7.) The constraint $B_{k} x \leqslant b_{k}$ is redundant in the system (1) if and only if the system

$$
\begin{aligned}
& A x=a, \\
& B_{i} x \leqslant b_{i}, \quad \forall i \neq k, \\
& B_{k} x>b_{k}
\end{aligned}
$$

is infeasible.

Some more relations between redundancy of a constraint and feasibility of a system of linear constraints can be obtained by dualizing the linear programming problem (2). As an example, we mention the following theorem. 
Theorem 2.2. (Ref. 1). The constraint $B_{k} x \leqslant b_{k}$ is redundant in the system (1) if and only if the system

$$
\begin{aligned}
& A^{T} w+B^{T} v=0, \\
& a^{T} w+b^{T} v \leqslant 0, \\
& v_{i} \geqslant 0, \quad \forall i \neq k, \\
& v_{k}=-1
\end{aligned}
$$

is feasible.

\section{Implicit Equalities}

Some inequalities may be replaced by equalities without enlarging the dimension (number of variables) of the system. Denote

$$
V_{k}=\left\{x \in R^{n} \mid B_{k} x=b_{k}\right\} \text {. }
$$

Then, we define implicit equalities as follows. ${ }^{3}$

Definition 3.1. The constraint $B_{k} x \leqslant b_{k}$ in the system (1) is an implicit equality if and only if

$$
S \subset V_{k}
$$

Denote

$$
\tilde{u}_{\mathrm{k}}=\max \left\{u_{k}(x) \mid x \in S_{k}\right\} \text {. }
$$

Then, we can prove the following theorem.

Theorem 3.1. The constraint $B_{k} x \leqslant b_{k}$ is an implicit equality in the system (1) if and only if

$$
\tilde{u}_{k}=0
$$

Proof. If Part. From

$$
\max \left\{u_{k}(x) \mid x \in S_{k}\right\}=0
$$

we see that

$$
B_{k} x \geqslant b_{k}, \quad \forall x \in S_{k} .
$$

\footnotetext{
${ }^{3}$ The concept of implicit equalities introduced here is similar to the concept of unstable inequalities (see, e.g., Ref. 10). In the present context, our terminology seems to be preferable.
} 
For all $x \in S$, we have

$$
B_{k} x \leqslant b_{k}
$$

together, this yields

$$
B_{k} x=b_{k},
$$

i.e.,

$$
x \in V_{k}, \quad \forall x \in S ;
$$

thus,

$$
S \subset V_{k} .
$$

Only If Part. If

$$
B_{k} x \leqslant b_{k}
$$

is an implicit equality, then

$$
u_{k}(x)=0, \quad \forall x \in S .
$$

Thus,

$$
\max \left\{b_{k}-B_{k} x \mid x \in S_{k}, B_{k} x \leqslant b_{k}\right\}=0,
$$

implying that

$$
\max \left\{u_{k}(x) \mid x \in S_{k}\right\}=\tilde{u_{k}}=0 .
$$

Note that, by Theorem 3.1, some similarity is shown between implicit equality constraints and redundant inequality constraints. Replacing the max operator in (4) by a min operator yields (2). Based on this observation, an algorithm to identify implicit equalities is derived from an algorithm to identify redundant inequality constraints (Ref. 1).

Again by dualizing the linear programming problem (4), we may obtain some theorems relating the feasibility of a system of linear constraints to an implicit equality. As an example, we mention the following theorem.

Theorem 3.2. (Ref. 1). The constraint $B_{k} x \leqslant b_{k}$ is an implicit equality in the system (1) if and only if the system

$$
\begin{aligned}
& A^{T} w+B^{T} v=-B_{k}, \\
& a^{T} w+b^{T} v=-b_{k}, \\
& v \geqslant 0
\end{aligned}
$$

is feasible. 
In the remainder of this section, we establish some relations between redundant constraints and implicit equalities in the system (1). We use the following notation:

$$
\begin{aligned}
& m=m_{a}+m_{b}, \\
& r=n-m_{a}, \\
& V=x \in R^{n} \mid A x=a .
\end{aligned}
$$

We denote by $\operatorname{lin}(S)$ the smallest linear manifold containing $S$, i.e., the subset of $R^{n}$ consisting of all linear combinations of vectors from $S$.

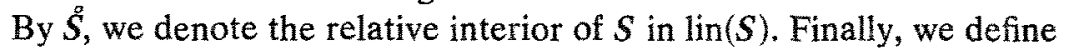

$$
\operatorname{dim} S=\operatorname{dim}(\operatorname{lin}(S))
$$

First, we prove three lemmas.

Lemma 3.1. If, in the system (1),

$$
\tilde{u}_{i}>0, \quad \forall i
$$

then

$$
S^{g}=\left\{x \in R^{\eta} \mid A x=a, B x<b\right\} .
$$

Proof. First, suppose that $x^{\prime} \in R^{n}$ is such that

$$
A x^{\prime}=a \text { and } B x^{\prime}<b .
$$

Then, around $x^{\prime}$, there is an $\epsilon$-ball $o\left(x^{\prime}, \epsilon\right)$ such that

$$
B z<b, \quad \forall z \in o\left(x^{\prime}, \epsilon\right) .
$$

Any

$$
x \in\left\{o\left(x^{\prime}, \epsilon\right) \cap \operatorname{lin}(S)\right\}
$$

is a linear combination of $x^{\prime}$ and elements from $S$, and so

$$
A x=a ;
$$

thus,

$$
z \in S \text {. }
$$

Therefore,

$$
\left\{o\left(x^{\prime}, \epsilon\right) \cap \operatorname{lin}(S)\right\} \subset S \text { and } \quad x^{\prime} \in \stackrel{S}{S} .
$$

For the remainder of the proof, it might be helpful to consider Fig. 1. 


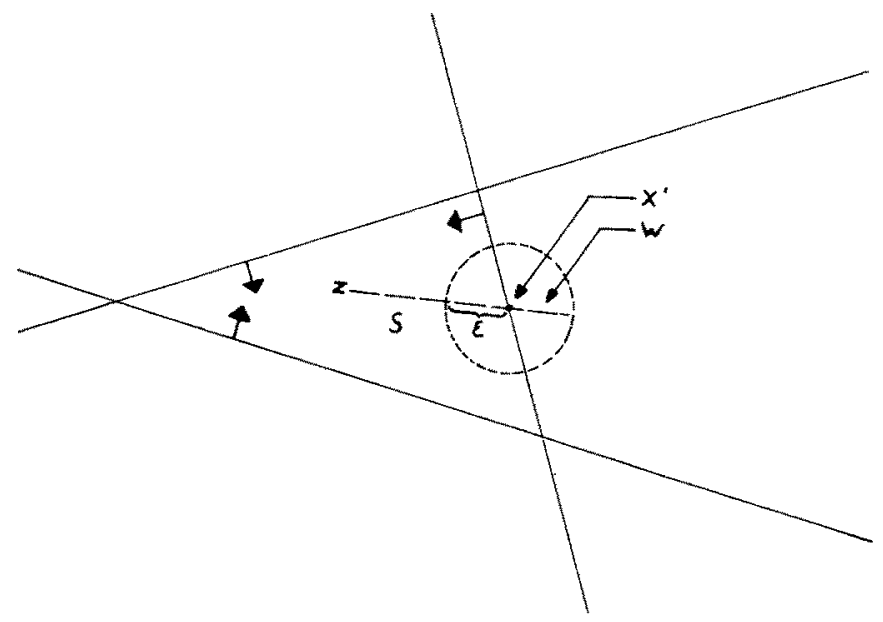

Fig. 1. Second part of the proof of Lemma 3.1.

Now, suppose that $x^{\prime} \in R^{n}$ is such that

$$
A x^{\prime}=a
$$

and

$$
B_{i} x^{\prime}<b_{i}, \quad \forall i=2, \ldots, m_{b}
$$

but

$$
B_{1} x^{\prime}=b_{1} \text {. }
$$

Since, in the system (1),

$$
\tilde{u}_{i}>0, \quad \forall i
$$

there is a point $z \in S$, with $B_{1} z<b_{1}$. We have to prove that there is a point $w$ in any $\left\{o\left(x^{\prime}, \epsilon\right) \cap \operatorname{lin}(S)\right\}$, with $w \notin S$. Choose

$$
w=\lambda x^{\prime}+(1-\lambda) z, \quad \text { with } \lambda=1+\epsilon .
$$

Then,

$$
\begin{aligned}
B_{1} w & =(1+\epsilon) B_{1} x^{\prime}-\epsilon B_{1} z \\
& =(1+\epsilon) b_{1}-\epsilon b_{1}=b_{1}
\end{aligned}
$$

thus, $w \notin S$.

Lemma 3.2. (Interior Point Lemma, Ref. 26.) If, in the system (1), $\tilde{u}_{i}>0, \forall i$, then $\dot{S} \neq \varnothing$. 
Proof. From

$$
\tilde{u}_{i}>0, \quad \forall i,
$$

we see that, for all $i$, there is a point $y^{i} \in S_{i}$ such that

\section{Consider}

$$
B_{i} y^{i}<b_{i}
$$

$$
y=\left(1 / m_{b}\right) \sum_{i=1}^{m_{b}} y^{i}
$$

It is easy to see that

$$
A y=a \text { and } B y<b \text {, }
$$

which means that

$$
y \in \stackrel{\circ}{S}
$$

Lemma 3.3. If the system (1) satisfies $r(A)=m_{a}$ and $\tilde{u}_{i}>0, \forall i$, then

$$
\operatorname{dim} S=n-m_{a}=r \text {. }
$$

Proof. From the interior point lemma, we know that there is a point $y \in S^{\circ}$ such that $\{V \cap o(y, \epsilon)\} \subset S$. Because of $r(A)=m_{a}$, we have

$$
\operatorname{dim}(V)=n-m_{a}=r ;
$$

therefore, for some basis $\left(\alpha_{1}, \ldots, \alpha_{r}\right)$, we can write

$$
V=y+\lambda_{1} \alpha_{1}+\cdots+\lambda_{r} \alpha_{r}, \quad\left(\lambda_{1}, \ldots, \lambda_{r}\right)^{T} \in R^{r} .
$$

Furthermore, $\exists \delta>0$ such that, if

$$
\left|\lambda_{i}\right| \leqslant \delta, \quad \forall i
$$

then

$$
\left\{y+\lambda_{1} \alpha_{1}+\cdots+\lambda_{r} \alpha_{r}\right\} \in o(y, \epsilon)
$$

and

$$
y, y+\delta \alpha_{1}, \ldots, y+\delta \alpha_{r} \in\{V \cap o(y, \epsilon)\} .
$$

Since $\delta \alpha_{1}, \ldots, \delta \alpha_{r}$ are linearly independent, we have

$$
\operatorname{dim}\{V \cap o(y, \epsilon)\} \geqslant r,
$$

which implies that

$$
\operatorname{dim} S \geqslant r \text {. }
$$


But, since $S \subset V$, we also have

$$
\operatorname{dim} S \leqslant r
$$

thus,

$$
\operatorname{dim} S=r .
$$

Using these lemmas, we can prove the following theorems, which apply to situations in which implicit equalities are explicitly stated as equalities. By definition, the set of feasible solutions $S$ does not change under such an operation.

Theorem 3.3. The system (1) in which $\tilde{u}_{i}>0, \forall i$ contains redundant equalities if and only if $r(A)<m_{a}$.

Proof. If Part. It follows from Corollary 2.1.

Only If Part. Suppose that

$$
r(\boldsymbol{A})=m_{a} \quad \text { and } \quad \tilde{u}_{i}>0, \quad \forall i .
$$

Then,

$$
\operatorname{dim} S=n-m_{a}
$$

(Lemma 3.3). If there are redundant equalities in the system, we can remove them, and the new system still has

$$
\tilde{u}_{i}>0, \quad \forall i
$$

and now satisfies

$$
r(A)=m_{a},
$$

too. Then, according to Lemma 3.3,

$$
\operatorname{dim} S=n-m_{a}^{\prime}>n-m_{a}^{\prime},
$$

and this contradicts the assumption.

Lemma 3.4. If the system (1) contains exactly one inequality constraint which is an implicit equality, then this inequality constraint is redundant as well.

Proof. Assume that the first inequality is an implicit equality,

$$
B_{1} x=b_{1}, \quad \forall x \in S .
$$

Then, the system

$$
B_{i} x \leqslant b_{i}, \quad i=2, \ldots, m_{b},
$$


contains no implicit equalities; hence, according to the interior point lemma, there is an $y \in S$ such that

$$
B_{i} y<b_{i}, \quad \forall i=2, \ldots, m_{b} .
$$

Suppose that the first inequality is not redundant. Then, there is a point $z \in S_{1}$, with

$$
B_{1} z>b_{1} \text {. }
$$

Take

$$
w(\lambda)=\lambda z+(1-\lambda) y .
$$

Since

we have

$$
o(y, \epsilon) \subset \mathfrak{S}_{1} \text { and } z \in S_{1} \text {, }
$$

$$
w(\lambda) \in S_{1}, \quad \text { for }-\frac{1}{2} \epsilon \leqslant \lambda \leqslant 1 \text {. }
$$

But

$$
B_{1} w\left(-\frac{1}{2} \epsilon\right)=-\frac{1}{2} \epsilon B_{1} z+\left(1+\frac{1}{2} \epsilon\right) B_{1} y<-\frac{1}{2} \epsilon b_{1}+\left(1+\frac{1}{2} \epsilon\right) b_{1}=b_{1} .
$$

Thus, the first inequality is not an implicit equality, contradicting the assumption.

This lemma is useful in proving the following theorem.

Theorem 3.4. If, in the system (1), all implicit equalities are replaced by equality constraints, then the new system contains at least one redundant equality.

Proof. Replacing an implicit equality

$$
B_{i} x \leqslant b_{i}
$$

by an equality constraint is equivalent to adding the constraint

$$
B_{i} x \geqslant b_{i}
$$

to the system. The latter constraint is obviously redundant. Furthermore, the last implicit equality is redundant by Lemma 3.4; and, if both

$$
B_{i} x \leqslant b_{i} \text { and } B_{i} x \geqslant b_{i}
$$

are redundant, the equality is redundant as well.

Finally, note that a strongly redundant constraint is not an implicit equality, and conversely. 


\section{Minimal Representation}

The set of feasible solutions $S$ for the system (1) may be represented in various ways, i.e., by different sets of linear constraints.

Definition 4.1. A minimal representation of the set $S$ is a system of linear constraints (1) with

$$
S=\left\{x \in R^{n} \mid A x=a, B x \leqslant b\right\},
$$

such that every other system describing $S$ has at least $m$ constraints.

In this section, we will give necessary and sufficient conditions for the system (1) to be a minimal representation. First we prove some lemmas.

Lemma 4.1. If the system (1) satisfies

$$
\begin{gathered}
r(\boldsymbol{A})=m_{a}, \\
\hat{u}_{i}<0, \quad \tilde{u}_{i}>0, \quad \forall i,
\end{gathered}
$$

then the system

$$
\begin{aligned}
A x & =a, \\
B_{1} x & =b_{1}, \\
B_{i} x & \leqslant b_{i}, \quad \forall i=2, \ldots, m_{b},
\end{aligned}
$$

contains no redundant equalities and implicit equalities.

Proof. Assume that the system (5) contains a redundant equality. Then, there is some $\lambda \in R^{m_{a}}$ such that

$$
B_{1}=\lambda_{1} A_{1}+\cdots+\lambda_{m_{a}} A_{m_{a}} .
$$

This yields, for all $x \in V$,

$$
B_{1} x=\lambda_{1} A_{1} x+\cdots+\lambda_{m_{a}} A_{m_{a}} x=\lambda_{1} a_{1}+\cdots+\lambda_{m_{a}} a_{m_{a}}=c,
$$

$c$ a constant; and this leads to a contradiction, since $b_{1} \geqslant c$ contradicts

$$
\hat{u}_{i}<0, \quad \forall i,
$$

and $b_{1} \leqslant c$ contradicts

$$
\tilde{u}_{i}>0, \quad \forall i,
$$

for the system (1). Therefore,

$$
r\left(\begin{array}{c}
A \\
B_{1}
\end{array}\right)=m_{a}+1
$$

and the system (5) contains no redundant equalities. 


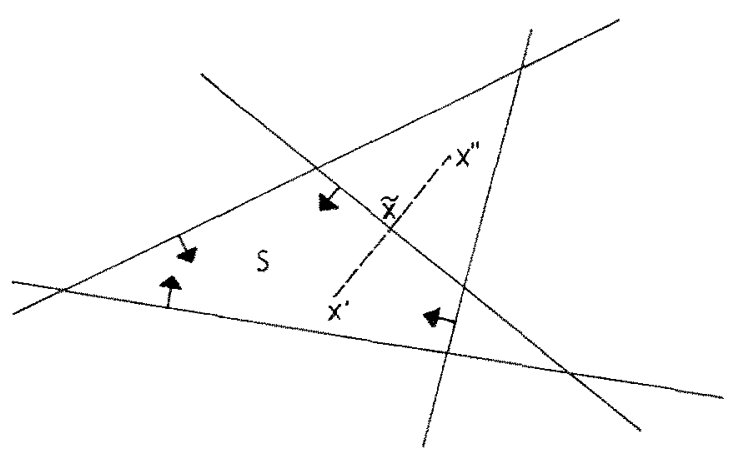

Fig. 2. Proving the second part of Lemma 4.1.

In following the proof that the system contains no implicit equalities, Fig. 2 might be helpful. According to the interior point lemma, there is some $x^{\prime} \in V$ with

$$
B_{i} x^{\prime}<b_{i}, \quad \forall i
$$

Since

$$
\tilde{u}_{i}>0, \quad \forall i,
$$

for the system (1) there is some $x^{\prime \prime} \in V$ with

$$
B_{1} x^{\prime \prime}>b_{1}
$$

and

$$
B_{i} x^{\prime \prime} \leqslant b_{i}, \quad i=2, \ldots, m_{b} .
$$

Choose $0<\lambda<1$ such that

$$
B_{1}\left(\lambda x^{\prime}+(1-\lambda) x^{\prime \prime}\right)=b_{1} .
$$

This is possible for

$$
\lambda=\left(b_{1}-B_{1} x^{\prime}\right) /\left(B_{1} x^{\prime \prime}-B_{1} x^{\prime}\right) .
$$

For all $2 \leqslant i \leqslant m_{b}$, we have

$$
B_{i}\left(\lambda x^{\prime}+(1-\lambda) x^{\prime \prime}\right)=\lambda B_{i} x^{\prime}+(1-\lambda) B_{i} x^{\prime \prime}<\lambda b_{i}+(1-\lambda) b_{i}=b_{i} .
$$

So

$$
\tilde{x}=\lambda x^{\prime}+(1-\lambda) x^{\prime \prime}
$$




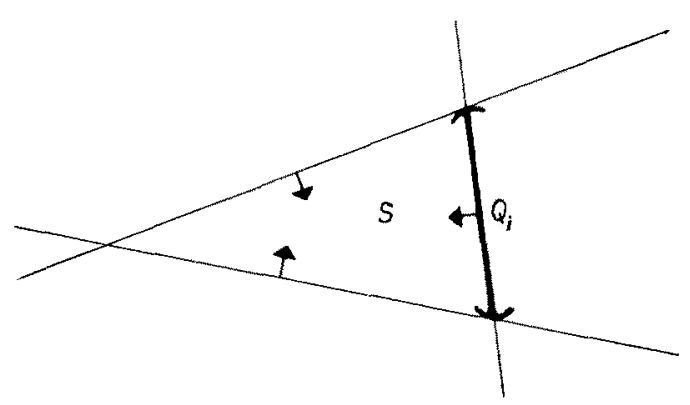

Fig. 3. Situation for Corollary 4.1 and Lemma 4.2 .

satisfies

$$
\begin{aligned}
& A \tilde{x}=a, \\
& B_{1} \tilde{x}=b_{1}, \\
& B_{i} \tilde{x}<b_{i}, \quad \forall i=2, \ldots, m_{b} ;
\end{aligned}
$$

therefore, the system (5) contains no implicit equalities.

Figure 3 might provide some help in studying the following corollary and the next lemma.

Corollary 4.1. If the system (1) satisfies

$$
\begin{gathered}
r(A)=m_{a}, \\
\hat{u}_{i}<0, \quad \tilde{u}_{i}>0, \quad \forall i,
\end{gathered}
$$

then for any $i \in\left(1, \ldots, m_{b}\right)$, there is a convex set $Q_{i}$ such that

$$
Q_{i} \subset\left(S_{i} \cap V_{i}\right) \text { and } \operatorname{dim} Q_{i}=r-1
$$

Proof. For all $x \in\left(S_{i} \cap V_{i}\right)$, we have

$$
\begin{aligned}
& A x=a, \\
& B_{i} x=b_{i}, \\
& B_{i} x \leqslant b_{j}, \quad \forall j \neq i .
\end{aligned}
$$

Take

$$
Q_{i}=S_{i} \cap V_{i}
$$

Then, clearly, $Q_{i}$ is convex. According to Lemma 4.1 the conditions for 
Lemma 3.3 are satisfied in this new system, and thus

$$
\operatorname{dim} Q_{i}=r-1 \text {. }
$$

Lemma 4.2. If, in the system (1),

$$
\tilde{u}_{i}>0, \quad \forall i,
$$

then for any convex set $Q$, with

$$
Q \subset\left(S \backslash S^{\sigma}\right) \text { and } \operatorname{dim} Q=r-1,
$$

there is some $V_{j}$ with

$$
Q \subset V_{j}
$$

Proof. Suppose that no $V_{i}$ contains $Q$. Then,

$$
\tilde{u}_{i}>0, \quad \forall i,
$$

implies that, for all $j$, there is an $y^{i} \in Q$ such that

$$
\begin{aligned}
& A y^{j}=a, \\
& B_{i} y^{j}<b_{j}, \\
& B_{i} y^{j} \leqslant b_{i}, \quad \forall i \neq j .
\end{aligned}
$$

Take

$$
y=\left(1 / m_{b}\right) \sum_{j=1}^{m_{b}} y^{i}
$$

Then, by the convexity of $Q$, we have $y \in Q$ and

$$
A y=a, \quad B y<b .
$$

Thus, $y \in \mathscr{S}$, contradicting the fact that

$$
Q \subset(S \backslash S) \text {. }
$$

Note that it may be proved that $Q \subset V_{j}$ for exactly one $j$; this, however, is not required for the following lemma.

Lemma 4.3. If, in the system (1),

$$
\tilde{u}_{i}>0, \quad \forall i,
$$

then

$$
V \not \subset V_{j}, \quad \text { for all } j .
$$


Proof. Suppose that there is some $j$ such that $V \subset V_{j}$. Then,

$$
B_{j} x=b_{j}, \quad \forall x \in V,
$$

implying that

$$
\max \left\{b_{j}-B_{j} x \mid x \in V\right\}=0 .
$$

But, since $S \subset V$, this yields

$$
\max \left\{b_{j}-B_{j} x \mid x \in S\right\} \leqslant 0,
$$

contradicting

$$
\tilde{u}_{i}>0, \quad \forall i
$$

Lemma 4.4. If the system (1) satisfies

$$
\begin{gathered}
r(\boldsymbol{A})=m_{a}, \\
\hat{u}_{i}<0, \quad \tilde{u}_{i}>0, \quad \forall i,
\end{gathered}
$$

then $V$ is the smallest linear manifold which contains both $Q_{k}$ and $Q_{j}$, $k \neq j$, with

$$
\begin{gathered}
Q_{k} \subset\left(V_{k} \cap S_{k}\right), \quad Q_{j} \subset\left(V_{j} \cap S_{j}\right), \\
\operatorname{dim} Q_{k}=\operatorname{dim} Q_{j}=r-1 .
\end{gathered}
$$

Proof. The linear manifold $V$ contains $Q_{k}$ and $Q_{j}$, by definition;

$$
\operatorname{dim} V=r \text {. }
$$

Suppose that there is another linear manifold $W$, containing $Q_{k}$ and $Q_{j}$; since

$$
\operatorname{dim} Q_{k}=\operatorname{dim} Q_{j}=r-1,
$$

we must have

$$
\operatorname{dim} W \geqslant r-1 .
$$

If

$$
\operatorname{dim} W=r \text { and } W \neq V \text {, }
$$

then

$$
\operatorname{dim}(V \cap W)=r-1,
$$

since

$$
(V \cap W)=W^{\prime}
$$


contains $Q_{k}$ and $Q_{j}$. Thus, $W^{\prime}$ would also be a linear manifold that contains both $Q_{k}$ and $Q_{j}$. Hence, we may restrict ourselves to the case

$$
\operatorname{dim} W=r-1 .
$$

Since both $W$ and $V_{k}$ are linear manifolds and $Q_{k} \subset V_{k}$, we have $W \subset V_{k}$; similarly, $W \subset V_{j}$. From the assumption, we have $W \subset V$; thus,

$$
W \subset\left(V \cap V_{k} \cap V_{j}\right) ;
$$

but, since

$$
\operatorname{dim}\left(V \cap V_{k}\right)=r-1
$$

(Lemma 3.3), this implies that

$$
\left(V \cap V_{k}\right) \subset V_{j} .
$$

The latter is impossible, because of the following (see also Fig. 4).

Choose some $x^{\prime} \in S^{g}$ (interior point lemma); thus,

$$
B_{i} x<b_{i}, \quad \forall i .
$$

Choose $x^{\prime \prime} \in S_{k}$ such that

$$
B_{k} x^{\prime \prime}>b_{k}
$$

which is possible according to

$$
\hat{u}_{i}<0, \quad \forall i .
$$

Then, there is some $0<\lambda<1$ with

$$
B_{k}\left(\lambda x^{\prime}+(1-\lambda) x^{\prime \prime}\right)=b_{k} .
$$

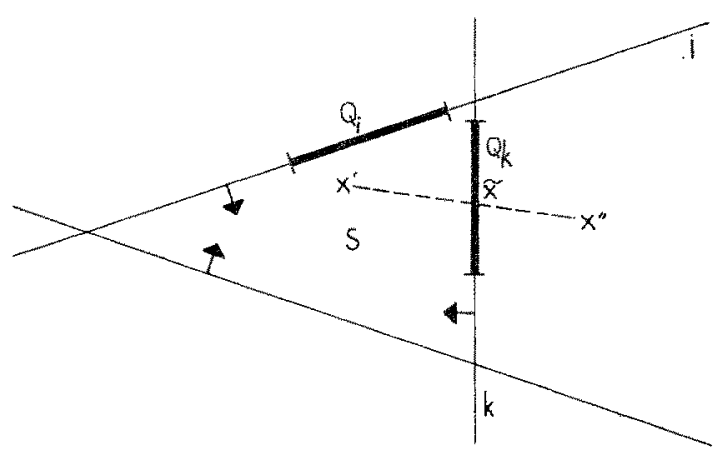

Fig. 4. Proving Lemma 4.4. 
Define

$$
\tilde{x}=\lambda x^{\prime}+(1-\lambda) x^{\prime \prime}
$$

since

$$
\tilde{x} \in V \text { and } \tilde{x} \in V_{k}
$$

also

$$
\tilde{x} \in\left(V \cap V_{k}\right)
$$

However,

$$
B_{j} \tilde{x}=\lambda B_{j} x^{\prime}+(1-\lambda) B_{j} x^{\prime \prime}<b_{j}+(1-\lambda) b_{j}=b_{j}
$$

therefore

$$
\tilde{x} \notin V_{j},
$$

thus

$$
\left(V_{k} \cap V\right) \not \subset V_{j} .
$$

At this point we are fully equipped to prove the main theorem.

Theorem 4.1. Minimal Representation Theorem. The system (1) is a minimal representation of the set $S$ if and only if it contains no redundant constraints and no implicit equalities.

Proof. Note that the system (1) contains no redundant constraints and implicit equalities if and only if

$$
\begin{gathered}
r(A)=m_{a}, \\
\hat{u}_{i}<0, \quad \forall i, \\
\tilde{u}_{i}>0, \quad \forall i .
\end{gathered}
$$

If Part. Suppose that there is another system (indicated by primes) that also represents $S$, but with $m^{\prime}<m$. We assume that

$$
\begin{gathered}
r\left(A^{\prime}\right)=m_{a^{\prime}}, \\
\hat{u}_{i}<0, \quad \forall i, \\
\tilde{u}_{i^{\prime}}>0, \quad \forall i,
\end{gathered}
$$

for that system as well.

First, we prove that the number of equalities in both systems is equal. Then, we prove that the sets of points satisfying all equality constraints 
are the same for both systems. According to Lemma 3.3,

$$
\operatorname{dim} S=n-n_{a}=r ;
$$

however, also

$$
\operatorname{dim} S=n-m_{a}^{\prime}=r,
$$

and therefore

$$
m_{a}=m_{a}^{\prime}
$$

The smallest linear manifold containing $S$ has dimension $n-m_{a}$, but also

$$
S \subset V \text { and } \operatorname{dim} V=n-m_{a} ;
$$

therefore, $V$ is the smallest linear manifold containing $S$. The same is true for $V^{\prime}$, and thus

$$
V^{\prime}=V^{\prime}
$$

Now, we show that every inequality constraint in one system corresponds to at least one inequality in the other system, but no two correspond to the same inequality. This implies that the number of inequality constraints in both systems is equal.

Choose

$$
i \in\left(1, \ldots, m_{b}\right) \text {; }
$$

Corollary 4.1 says that there is some

$$
Q_{i} \subset\left(S_{i} \cap V_{i}\right),
$$

for this $i$; and according to Lemma 4.2, there is some $V_{i^{\prime}}$ corresponding to this $Q_{i}$. Thus, related to any $i$, there is an $i^{\prime}$. Suppose now that, for $i \neq j$, we have $i^{\prime}=j^{\prime}$, thus

$$
V_{i^{\prime}}=V_{i^{\prime}}
$$

thus

$$
Q_{i} \subset V_{i^{\prime}} \text { and } Q_{j} \subset V_{i^{\prime}}
$$

but, according to Lemma 4.4 , the smallest linear manifold containing both $Q_{i}$ and $Q_{j}$ is $V$. Therefore, $V \subset V_{i^{\prime}}$ which would be contradicting Lemma 4.3. Thus, for $i \neq j$, we have $i^{\prime} \neq j^{\prime}$, and therefore

$$
m_{b} \leqslant m_{b}^{\prime} \text {. }
$$

In the same way, we prove that

$$
m_{b}^{\prime} \leqslant m_{b}
$$


and thus

$$
m_{b}=m_{b}^{\prime}
$$

Only If Part. Suppose that the system (1) contains redundant constraints; these may be removed to obtain a smaller system. This contradicts the fact that the system is a minimal representation. Suppose that the system (1) contains implicit equations; replacing all of them by equality constraints would make at least one of them redundant (Theorem 3.4), so this one may be removed to obtain a smaller system. Again, this is a contradiction to the minimality of the representation.

It is worthwhile to mention that, in fact, we have proved a slightly stronger theorem: not only the minimal number of constraints is uniquely determined, but also the number of equalities and inequalities in the minimal representation is unique. But, even though these numbers are unique, the minimal representation in itself is not unique.

At this point, we should stress two important features of the minimal representation theorem: given the knowledge of which constraints are redundant and of which equalities are implicit, no transformations of the system are necessary to obtain the minimal representation. Even more important is the fact that the theorem implicitly guarantees that every reduction of the system is a step in the right direction. It is not possible to get a locally minimal representation by a specific order in which redundant constraints and implicit equalities are removed.

\section{Relation with Existing Theory}

Existing theory on the representation of a convex polyhedral set can be shown to arise as a special case of the result in the previous section. First, we consider the work of Shefi (Ref. 11), modified and extended by Luenberger (Ref. 12).

Definition 5.1. (Refs. 11 and 12). A minimal similar representation for the convex polyhedral set $T$ is a system of linear constraints

$$
D x=d, \quad x \leqslant 0,
$$

with

$$
D \in R^{m_{d} \times n_{d}}, \quad x \in R^{n_{d}}, \quad 0 \in R^{n_{d}}, \quad d \in R^{n_{d}},
$$

such that:

(i) for

$$
\bar{T}=\left\{x \in R^{n d} \mid D x=d, x \geqslant 0\right\},
$$


there is a linear invertible mapping $\Sigma$, called a similarity transformation, that maps $\operatorname{lin}(T)$ onto $\operatorname{lin}(\bar{T})$, such that

$$
\Sigma(T)=\bar{T} \quad \text { and } \quad \Sigma^{-1}(\bar{T})=T
$$

(ii) $m_{d}$ is minimal;

(iii) $n_{d}$ is minimal.

Loosely speaking, this means that a minimal similar representation of a set $T$ is a system of linear equalities in nonnegative variables that determines a set $\bar{T}$ with the same shape and dimensions as $T$ and does this in the smallest number of equalities and the smallest number of variables.

The main difference between a minimal representation and a minimal similar representation is that the latter is embedded in a linear manifold of smallest possible dimension and is given relative to that linear manifold.

Shefi and Luenberger introduce the following terminology:

Null variables are defined to be zero in every feasible solution; in our terminology, this means that the corresponding inequality constraint is an implicit equality. Null variables can be removed by striking out the corresponding column in the matrix $D$ and adding the equality $x_{j}=0$.

Nonextremal variables are those variables such that the corresponding nonnegative constraint $x_{i}=0$ is redundant. Nonextremal variables can be removed from the system by eliminating them from a constraint in which they have a nonzero coefficient. Then, this constraint can be replaced by a definition of the removed variable in terms of the other variables; e.g., if $x_{1}$ is nonextremal and $a_{11} \neq 0$, then the constraint

$$
\sum_{j=1}^{n} a_{1 j} x_{j}=b_{1}
$$

may be replaced by

$$
x_{1}=\left(1 / a_{11}\right)\left(b_{1}-\sum_{j=2}^{n} a_{1 j} x_{j}\right)
$$

Now, we can prove the following theorem.

Theorem 5.1. For $T \neq \varnothing$, the system (6) is a minimal similar representation of $T$ if and only if it contains no redundant equations, null variables, and nonextremal variables. 
Proof. If Part. If the system (6) contains no redundant equations, null variables, or nonextremal variables, we have

$$
\begin{gathered}
r(D)=m_{d}, \\
\hat{u}_{i}<0, \quad \forall i, \\
\tilde{u}_{i}>0, \quad \forall i ;
\end{gathered}
$$

so, we can apply the main redundancy theorem; therefore, the system (6) is a minimal representation.

We have proved that the number of faces of $T$ of dimension equal to $\operatorname{dim} T-1$ is fixed and equal to the number of inequality constraints. Since the number of these faces does not change under a similarity transformation, we cannot transform $T$ to a space with smaller dimension. Since we also proved that

$$
\operatorname{dim} T=n_{d}-m_{d}
$$

and hence is constant, we cannot find a system with less equations in the same number of variables.

Only If Part. Assume that the systern (6) is a minimal similar representation. If it contains a redundant equality, this can be dropped, contradicting point (ii) of the definition of a similar representation. If it contains a null variable, point (iii) would be contradicted. It it contains a nonextremal variable $x_{k}$, points (ii) and (iii) are contradicted.

Shefi and Luenberger proved this theorem under the additional assumption that $T$ is bounded (Refs. 11 and 12). However, as shown here, by using our result on minimal representations, the theorem holds for more general cases too. Therefore, the result of Shefi and Luenberger follows immediately from Theorem 5.1.

The minimal similar representation of a set $T$ may seem to be smaller than the minimal representation, but this is an illusion. In fact, this constitutes an important point of criticism on the theory of Shefi and Luenberger.

In the minimal similar representation, a minimal dimension (number of variables) is required: variables corresponding to dimensions that are dropped are appended to the system as equalities. Their values can be calculated afterward. However, this is no reason to consider these variables as not being a part of the system. From a practical point of view, there is no such thing as lowest dimension: all variables specified in the set $T$ should be present in a minimal representation as well. Therefore, the variables that are appended to the system, in a minimal similar representation, form an integral part of the system and should also be incorporated in the minimal representation. 
Another way to interpret this point is to consider the minimal similar representation as a one-to-one transformation of the original system. Given the minimal similar representation, nothing can be said about the original system if the transformation is not known. Therefore, the minimal similar representation is useless without this one-to-one transformation. This implies that, since the transformation is embedded in the equalities that are appended to the system, these equalities should be part of a minimal representation, as is done in our theory.

A minimal similar representation plus the equalities appended to the system will never be smaller than our minimal representation. Furthermore, our reductions to obtain a minimal representation require no additional calculations (after identification of the constraints), whereas in the theory of Shefi and Luenberger the removal of nonextremal variables requires extra calculations for all the coefficients in the system.

Eckhardt (Ref. 13) considers systems in which only linear inequality constraints are present. By defining unstable inequalities in the same way as our implicit equalities, Eckhardt can prove a number of special cases $\left(m_{a}=0\right)$ of our theorems and lemmas.

Assuming that there are no unstable inequality constraints, Eckhardt proceeds by defining a minimal representation as a system that contains no redundant constraints. Since he addresses the case $m_{a}=0$, his notion of a minimal representation coincides with the one developed here. However, instead of proving the minimality of the number of constraints, Eckhardt simply defines a minimal representation in the way sketched above. Thus, he avoids a confrontation with the minimal representation theorem, even for the simple case in which

$$
m_{a}=0 \text { and } \tilde{u}_{i}>0, \quad \forall i \text {. }
$$

\section{References}

1. Telgen, J., Redundancy and Linear Programs, Erasmus University, Rotterdam, Holland, PhD Thesis, 1979.

2. DantzIG, G. B., Linear Programming and Extensions, Princeton University Press, Princeton, New Jersey, 1963.

3. FARKAS, J., Über die Theorie der Einfachen Ungleichungen, Journal für Reine und Angewandte Mathematik, Vol. 124, pp. 1-27, 1902.

4. MotzKIN, T. S., Beiträge zur Theorie der Linearen Ungleichungen, Basel University, PhD Thesis, 1936.

5. KUHN, H. W., and TUCKER, A. W., Editors, Linear Inequalities and Related Systems, Princeton University Press, Princeton, New Jersey, 1956.

6. TSCHERNIKOW, S. M., Lineare Ungleichungen, VEB Deutscher Verlag der Wissenschaften, Berlin, Germany, 1966 (translated by H. Weinert).

7. Boot, J. C. G., On Trivial and Binding Constraints in Programming Problems, Management Science, Vol. 8, pp. 419-441, 1962. 
8. Charnes, A., Cooper, W. W., and Thompson, G. L., Some Properties of Redundant Constraints and Extraneous Variables in Direct and Dual Linear Programming Problems, Operations Research, Vol. 10, pp. 711-723, 1962.

9. ThOMPSON, G. L., TONGE, F. M., and ZIONTS, S., Techniques for Removing Nonbinding Constraints and Extraneous Variables from Linear Programming Problems, Management Science, Vol. 12, pp. 588-608, 1966.

10. RobInson, S. M., Stability Theory for Systems of Inequalities, SIAM Journal of Numerical Analysis, Vol. 12, pp. 754-769, 1975.

11. SHEFI, A., Reduction of Linear Inequality Constraints and Determination of All Feasible Extreme Points, Stanford University, PhD Thesis, 1969.

12. Luenberger, D. G., Introduction to Linear and Nonlinear Programming, Addison-Wesley, Reading, Massachusetts, 1973.

13. ECKHARDT, U., Semidefinite Lineare Komplementärprobleme, RWTH Aachen, Habilitationsschrift, 1977.

14. ECKHARDT, U., Representation of Convex Sets, Proceedings of the International Symposium on Extremal Methods and Systems Analysis, Austin, Texas, 1979.

15. LLEWELlyn, R. W, Linear Programming, Holt, Rinehart, and Winston, New York, NY, 1964.

16. ZIONTS, S., Size Reduction Techniques of Linear Programming and Their Application, Carnegie Institute of Technology, $\mathrm{PhD}$ Thesis, 1965.

17. ECKHARDT, U., Redundante Ungleichungen bei Linearen Ungleichungssysteme, Unternehmensforschung, Vol. 12, pp. 279-286, 1971.

18. MAtTHeIS, T. H., An Algorithm for Determining Irrelevant Constraints and All Vertices in Systems of Linear Inequalities, Operations Research, Vol. 21, pp. 247-260, 1973.

19. GAL, T., Zur Identifikation Redundanter Nebenbedingungen in Linearen Programmen, Zeitschrift für Operations Research, Vol. 19, pp. 19-28, 1975.

20. GAL, T., Redundancy in Systems of Linear Inequalities Revisited, Fernuniversität Hagen, Working Paper No. 19, 1978.

21. BONEH, A., and GOLAN, A., Constraints Redundancy and Feasible Region Boundedness by Random Feasible Point Generator, Technion, Haifa, Israel, Technical Report, 1979.

22. ZIMMERMAN, H. J, and GAL, T., Redundanz und Ihre Bedeutung für Betrie bliche Optimierungsentscheidungen, Zeitschrift für Betriebswirtschaft, Vol. 45, pp. 221-236, 1975.

23. SPRonk, J., and Telgen, J., A Note on Multiple Objective Programming and Redundancy, Erasmus University, Rotterdam, Holland, Center for Research in Business Economics, Report No. 7906, 1979.

24. TELGEN, J., Overbodige en Niet-Bindende Restricties in Lineare Programmerings Problemen, Bedrijfskunde, Vol. 51, pp. 168-173, 1979.

25. KARWAN, M. H., TELGEN, J., and ZIONTS, S., Redundancy in Mathematical Programming, Springer Verlag, New York, New York, 1982.

26. ECKHARDT, U., Theorems on the Dimension of Convex Sets, Linear Algebra and Its Aplications, Vol. 12, pp. 63-76, 1975. 